\title{
Carbapenemasas en bacterias Gram negativas no fermentadoras aisladas en servicios críticos del Hospital Regional Lambayeque, diciembre 2014 - julio 2015
}

\section{Carbapenemases in non-fermenting Gram-negative bacteria isolated in critical care services from the Lambayeque Regional Hospital, December 2014 - July 2015}

Correspondencia Rosy M. Gastelo-Acosta rosy_gastelo_15@hotmail.com

\footnotetext{
Recibido: 16/08/2016

Arbitrado por pares

Aprobado: 14/0g/2016

Citar como: Gastelo-Acosta RM, Díaz-Sipión RS, Maguiña Vargas C. Carbapenemasas en bacterias Gram negativas no fermentadoras aisladas en servicios críticos del Hospital Regional de Lambayeque, diciembre 2014-julio 2015. Acta Med Peru. 2016;33(3):183-8
}

Rosy M. Gastelo-Acosta' ${ }^{1}$, Roberto S. Díaz-Sipión², Ciro Maguiña Vargas ${ }^{3,4}$

1 Facultad de Ciencias Biológicas, Universidad Nacional Pedro Ruiz Gallo. Lambayeque, Perú.

2 Hospital Regional de Lambayeque. Lambayeque, Perú.

3 Vicerrectorado de Investigación, Universidad Peruana Cayetano Heredia. Lima, Perú.

4 Departamento de Enfermedades Infecciosas Tropicales y Dermatológicas, Hospital Nacional Cayetano Heredia. Lima, Perú.

\section{RESUMEN}

Objetivo. Determinar la presencia de bacterias Gram negativas no fermentadoras productoras de carbapenemasas de los servicios de cuidados críticos y emergencias del Hospital Regional Lambayeque, diciembre 2014 - julio 2015. Material y métodos: Se incluyó todos los aislamientos de bacterias Gram negativas no fermentadoras de muestras clínicas en los servicios seleccionados. La detección de carbapanemasas se realizó en dos pasos, primero se selecciónó a los sospechosos usando el método Kirby Baver con ellos se aplicaron tres métodos, la aproximación de de discos, Hodge modificado y Blue Carba. Resultados: Se analizó 50 asilamientos bacterianos de muestras de secreciones y líquidos provenientes de pacientes con diagnóstico presuntivo de infección, $48 \%$ presentó carbapanemasas. Todas las cepas de Acinetobacter baumannii 21/21 presentaron carbapanemasas tipo oxacilinasas y $3 / 29$ cepas de Pseudomonas aeruginosa presentaron carbapanemasas tipo metalobetalactamasas. Conclusiones: Casi la mitad de cepas aisladas producen carbapanemasas. La vigilancia de estas cepas y una política para el control de la resistencia antimicrobiana son necesarias de trabajar para evitar la expansión de este problema.

Palabras clave: Bacterias gramnegativas, farmacorresistencia microbiana, acinetobacter, pseudomonas. (Fuente: DeCS BIREME) 


\begin{abstract}
Objective: To determine the presence of carbapenemase producing non-fermenting Gram negative bacteria in critical care and emergency services from the Lambayeque Regional Hospital, December 2014 - July 2015. Material and methods: We included all isolates of non-fermenting Gram negative bacteria from clinical samples in the aforementioned services. Carbapenemase detection was performed in a two-step fashion: first, suspected samples were selected using the Kirby Bauer method. These samples underwent three further assessments, double disk approximation, modified Hodge, and Blue Carba tests. Results: Fifty bacterial isolates from secretion and fluid samples taken from patients with a presumptive diagnosis of infectious conditions were analyzed, and $48 \%$ of these samples were positive for the presence of carbapenemases. All Acinetobacter baumanii strains (21/21) had oxacillin-type carbapenemases and 3/29 Pseudomonas aeruginosa strains had metallo-betalactamase carbapenemases. Conclusions: Almost half of the isolated strains produced carbapenemases. It is necessary to perform surveillance activities on these strains and to have a firm policy for controlling antimicrobial resistance in order to prevent the expansion of these conditions.
\end{abstract}

Key words: Gram-negative bacteria, drug resistance, microbial, acinetobacter, pseudomonas (Source: MesH NLM)

\section{INTRODUCCIÓN}

La resistencia bacteriana es un fenómeno creciente caracterizado por la capacidad natural o adquirida de una cepa bacteriana a permanecer refractaria a los efectos bactericidas o bacteriostáticos de un antimicrobiano. El uso indiscriminado de los antibióticos y la presión selectiva ambiental, realizada por los antisépticos y desinfectantes, ha generado una respuesta de supervivencia en los microorganismos, que los capacita para evadir eficazmente la acción bactericida de los antimicrobianos ${ }^{[1-3]}$.

En marzo del 2014 la Organización Mundial de la Salud, Organización Panamericana de la Salud (OMS/OPS) subraya la importancia de reforzar la vigilancia y establecer estrategias de control para prevenir la diseminación de microorganismos con mecanismos de resistencia tipo "New Delhi metalobetalactamasa" (NDM). Desde 2008 , se ha documentado a nivel mundial la circulación de este tipo de microorganismos, un tipo de carbapenemasas que ocasiona resistencia a todos los antibióticos betalactámicos excepto aztreonam.

En el Perú el primer caso de carbapenemasas tipo KPC (Klebsiella pneumoniae productora de carbapenemasas) fue registrado el 11 de Octubre del 2013 en el Laboratorio de Microbiología del Hospital Nacional Arzobispo Loayza, la muestra procesada fue de un hemocultivo de un paciente de la Unidad de Cuidados Intensivos $(\mathrm{UCl})^{[2]}$. Es muy probable que se haya diseminado en los diferentes hospitales de las regiones del país; las bacterias Gram negativas no fermentadoras que se han registrado en los últimos meses en el Hospital Regional Lambayeque (HRL) son Acinetobacter baumannii y Pseudomonas aeruginosa (registros de datos bacterianos - HRL) y suelen presentar resistencia a los antibióticos betalactámicos y a otras familias de antibióticos.

En el HRL aún no se ha investigado la presencia las enzimas carbapenemasas, pero, se presume que ya están presentes en los diferentes servicios de atención, una de las razones es por la ineficacia de los antibióticos administrados en los pacientes, generando así la preocupación e inquietud del personal de salud, por tal motivo surge la necesidad colectiva de dar inicio a las investigaciones respectivas para su determinación y así tomar las medidas para evitar la diseminación de estas cepas en el ambiente hospitalario y tomar la mejor decisión en cuanto a la administración de los antibióticos.

El objetivo del estudio fue determinar la presencia de bacterias Gram negativas no fermentadoras productoras de carbapenemasas de los servicios de cuidados críticos y emergencias del Hospital Regional Lambayeque.

\section{MATERIAL Y MÉTODOS}

Se realizó un estudio transversal en el periodo de diciembre 2014 a julio 2015, se incluyó a todas las cepas aisladas de cultivos clínicos de los servicios de cuidados críticos ( $U C \mathrm{Cl}$, unidad de cuidados intermedios [UCIN] y UCI neonatal [UCI-NEO]) y emergencias del $\mathrm{HRL}$, luego se excluyó a aquellas bacterias que no fueran Gram negativas no fermentadoras.

Posteriormente usando el método de Kirby Bauer (CLSI 2015) [4] se seleccionó a las cepas sospechosas de producir carbapenemasas. Para Pseudomona incluyó la realización de antibiogramas utilizando los siguientes discos de antibióticos: ceftazidima, amoxicilina ácido clavulámico, cefepime, amikacina, gentamicina, ciprofloxacino, piperacilinatazobactam, aztreonam, imipenem, meropenem, y colistín, teniendo en cuenta principalmente el halo de meropenem $\leq 23 \mathrm{~mm}$ y ceftazidima $\leq 22 \mathrm{~mm}$ para considerar una cepa sospechosa de producir carbapenemasas.

En el caso de Acinetobacter baumannii se utilizaron los siguientes discos de antibióticos: ceftazidima, amoxicilina ácido clavulámico, cefepime, amikacina, gentamicina, ciprofloxacino, piperacilinatazobactam, imipenem, meropenem, y colistín, y fueron consideradas cepas sospechosas de carbapenemasas a aquellas que presentaron un halo $\leq 21 \mathrm{~mm}$ para imipenem. 


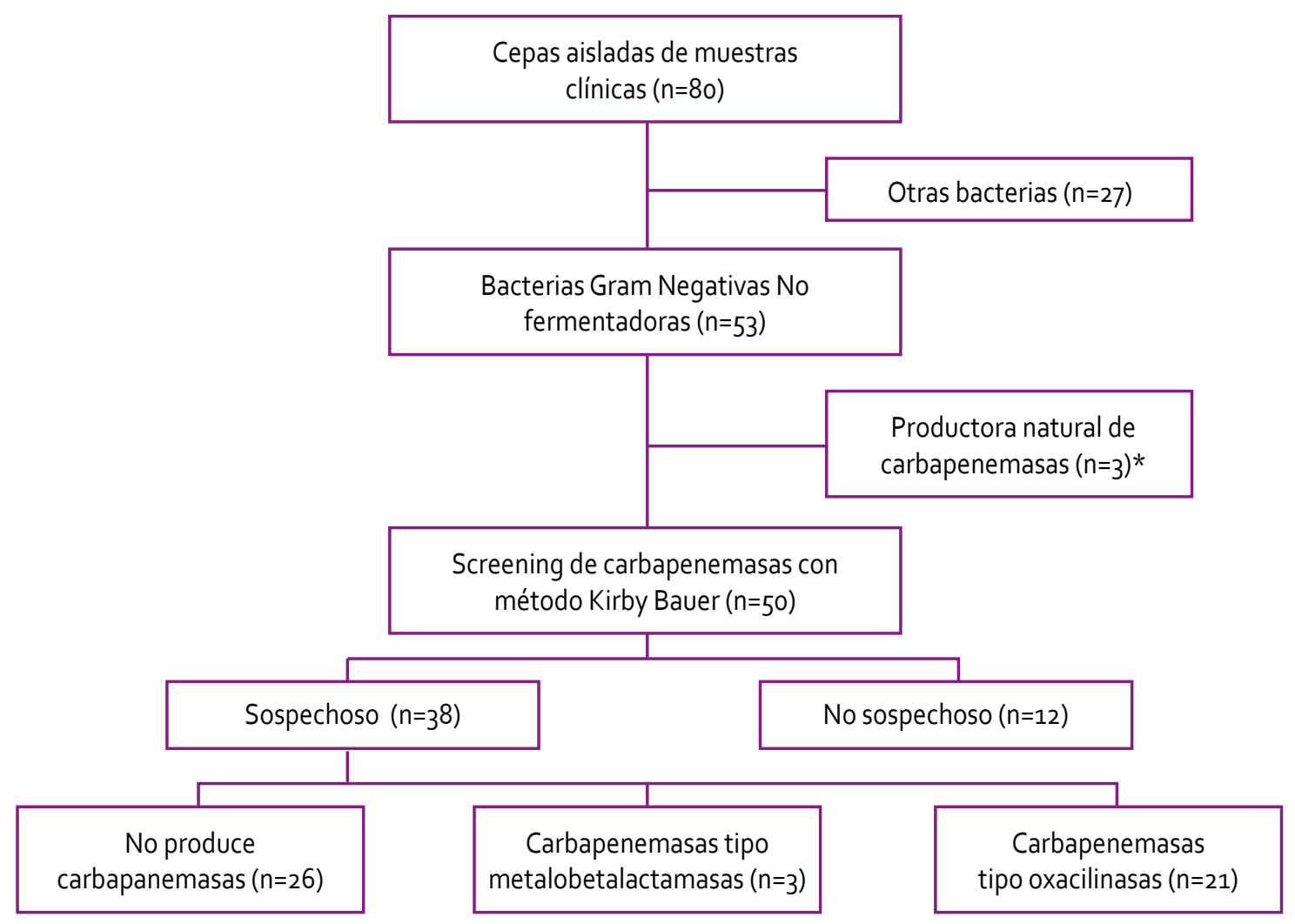

Figura 1. Flujograma para la detección de carbapanemasas en muestras clínicas de servicios críticos y emergencias del Hospital Regional de Lambayeque, diciembre 2014 - julio 2015.

* Stenotrophomonas maltophilia presenta naturalmente la enzima carbapenemasa.

Para la determinación de carbapenemasas en las cepas sospechosas se utilizaron tres métodos: aproximación de discos ${ }^{[5]}$, Hodge modificado (CLSI 2015) ${ }^{[4]}$ y Blue Carba ${ }^{[6]}$, para la prueba de aproximación de discos se utilizó solamente el disco de EDTA, el cual detecta solamente carbapenemasas tipo metalobetalactamasas; Hodge modificado detecta tres tipos de carbapenemasas (metalobetalactamasas, Kpc y oxacilinasas); y el test Blue Carba, determina tres tipos de carbapenemasas según el tiempo de reacción.

La información obtenida fue registrada y compilada en una base de datos utilizando el programa Microsoft Excel 2013. Los datos fueron expresados en frecuencias relativas y absolutas, se evaluó asociación entre la presencia de carbapenemasas según servicio, bacteria usando la prueba exacta de Fisher. Se consideró un $p$ $<0,05$ como significativo.

\section{RESULTADOS}

\section{Bacterias Gram negativas no fermentadoras}

El total de cepas aisladas en este estudio fueron 80 , de las cuales 53 (66\%) correspondieron a bacterias Gram negativas no fermentadoras, la mayoría de $\mathrm{UCl}$ 43/53 y ninguna del servicio de emergencias. Los aislamientos fueron más frecuentes en personas mayores de 60 años $(25 / 53,41 \%)$ y procedieron principalmente de muestras de secreción bronquial $(41 / 53,77 \%)$, aunque también de hemocultivos, líquido cefalorraquídeo, etc (Figura 1).

\section{Producción de carbapenemasas}

De los 53 cultivos de bacterias gram negativas no fermentadoras, solo se evaluó la presencia de carbapenemasas en 50 de ellas, ya que las tres restantes correspondieron a Stenotrophomonas maltophilia y naturalmente presentan la enzima carbapenemasa. La frecuencia de producción de carbapenemasas fue de $48 \%$ (24/50), la mayoría de estas procedieron de $\mathrm{UCl}(21 / 24,87 \%)$.

De las 24 cepas productoras de carbapenemasas, la mayoría fueron aisladas de adultos mayores (15/24, 62\%). Todos los aislamientos de Acinetobacter baumannii fueron productores de carbapenemasas y representaron el $87 \%$ de los casos y $3 / 26$ (10\%) de los aislamientos de Pseudomonas aeruginosa lo presentaron. La mayoría de cepas productoras de carbapenemasas fueron aisladas de muestras de secreción bronquial (20/24, 83\%), seguido de punta de catéter $(3 / 24,13 \%)$. No se encontró asociación entre las características medidas y la presencia de carbapenemasas $(p>0,05)$ excepto con el tipo de bacteria (Tabla 1). 
Tabla 1. Cepas productoras de carbapenemasas aisladas de servicios críticos y emergencias del Hospital Regional de Lambayeque, diciembre 2014 - julio 2015.

\begin{tabular}{|c|c|c|c|c|c|c|c|}
\hline & \multirow{2}{*}{\multicolumn{2}{|c|}{ Total }} & \multicolumn{4}{|c|}{ Cepas productoras de carbapenemasas } & \multirow{3}{*}{ p* } \\
\hline & & & \multicolumn{2}{|c|}{ Sí } & \multicolumn{2}{|c|}{ No } & \\
\hline & n & $(\%)$ & $\mathbf{n}$ & $(\%)$ & n & $(\%)$ & \\
\hline \multicolumn{8}{|l|}{ Servicio } \\
\hline $\mathrm{UCl}$ & 40 & (80) & 21 & $(52)$ & 19 & $(48)$ & 0,729 \\
\hline UCIN & 8 & (16) & 3 & (37) & 5 & $(63)$ & \\
\hline UCI-NEO & 2 & $(4)$ & 0 & (0) & 2 & (100) & \\
\hline Emergencias & 0 & (0) & 0 & (0) & 0 & (0) & \\
\hline \multicolumn{8}{|l|}{ Bacteria } \\
\hline A. baumannii & 21 & $(42)$ & 21 & $(100)$ & 0 & (0) & $<0,001$ \\
\hline$P$. aeruginosa & 29 & (58) & 3 & (10) & 26 & (90) & \\
\hline
\end{tabular}

UCI: Unidad de cuidados Intensivos, UCIN: Unidad de cuidados intermedios, UCI-NEO: Unidad de cuidados intensivos neonatales. * prueba exacta de Fisher

\section{Tipos de carbapenemasas}

Las cepas de Pseudomonas aeruginosa presentaron carbapenemasas tipo metalobetalactamasas (3/3) y Acinetobacter baumannii carbapenemasas tipo oxacilinasas (21/21). El método de aproximación de discos solo detectó las carbapenemasas de las ceptas de $P$. aeruginosa por ser metalobetalactamasas. Con el método Hodge se detectó $23 / 24$ casos y con el método Blue Carba $24 / 24$, con estos métodos se pudo determinar que las carbapenemasas del A. baumannii era tipo OXA.

\section{DISCUSIÓN}

El presente estudio pone de manifiesto el problema de la multiresistencia en los aislamientos de Acinetobacter baumannii y Pseudomonas aeruginosa identificados en el Hospital Regional Lambayeque - Perú. El panorama no es alentador al comparar los resultados obtenidos con otros estudios en el caso de Acinetobacter baumannii ${ }^{[7]}$. Con respecto a Pseudomonas aeruginosa los resultados se asemejan a los obtenidos en otras investigaciones nacionales e internacionales ${ }^{[8-10]}$.

De las 53 cepas aisladas, la mayoría provinieron de los servicios de UCl, 43 aislamientos, debido a factores predisponentes tales como inmunosupresión, conexión a equipos de ventilación mecánica, catéteres, sondas, y la estancia hospitalaria prolongada. Difiriendo con los resultados obtenidos en un estudio realizado en el mismo hospital en el cual identificaron 102 cepas de bacterias gram negativas no fermentadoras de los servicios UCl y UCIN del Hospital Regional Lambayeque, febrero - julio 2014, esto implica una reducción en el menor número de aislamientos para el periodo de este estudio ${ }^{[11]}$.
Se observó que la mayoría de cepas aisladas correspondían al grupo etario de 60 años a más, coincidiendo con el trabajo realizado el mismo año y en el mismo hospital con un $35 \%$ [5]. No se ha descrito el por qué existe la mayor probabilidad de aislar cepas bacterianas en pacientes adultos mayores, pero asumimos que es por la edad ya que muchos ingresan con enfermedades de base que favorecen al mal funcionamiento sistémico.

Así mismo de las 53 cepas, el $77 \%$ procedieron de muestras de secreciones bronquiales, estos hallazgos son muy similares al estudio de Menéndez en Cuba ${ }^{[2]}$. En el presente estudio el tipo de muestra procesada con mayor frecuencia fue las de secreción bronquial, ello explica el mayor porcentaje obtenido de bacterias Gram negativas no fermentadoras en muestra de secreción bronquial.

La frecuencia de $P$. aeruginosa productoras de carbapenemasas se encuentra en el rango de lo reportado en otros estudios. En el Instituto Nacional de Salud del Niño en el año 2013 se obtuvo $15,7 \%$ de cepas productoras de 51 aislamientos, estos se evaluaron con criterios según CLSI-2015, por ensayo fenotípico con método de aproximación de discos (imipenem y meropenem) y con ácido etilendiaminotetraacético (EDTA). Un estudio previo en el Hospital Nacional Guillermo Almenara Irigoyen en el 2008, se evaluó 186 cepas provenientes del servicio de microbiología, de las cuales 13 cepas fueron positivas para metalobetalactamasas correspondientes al 7,0\% ${ }^{[8-9]}$. Con ello se observa un potencial aumento de bacterias productoras de carbapenemasas del 2008 al 2015, aunque en este estudio se trabajó con muestras de servicios críticos, que es donde hay más riesgo de presentar cepas resistentes. Sin embargo, este incremento de la resistencia es posible continúe ocurriendo, pues las enzimas se trasmiten y se diseminan fácilmente, en 
este caso por las porinas específicas de recepción del pili sexua denominado OmpA. Por otro lado

Todas las cepas de A. baumannii presentaron carbapenemasas, esta resistencia a carbapenemes en $A$. baumannii está esencialmente asociada a la producción de enzimas OXA (betalactamasas de clase $D$ de Ambler), y con menor frecuencia a la producción de metalobetalactamasas ${ }^{\left[{ }^{[3]}\right.}$. Debido a la facilidad de la especie para captar y expresar nuevos mecanismos de resistencia, no es sorprendente que también se haya descrito carbapenemasas tipo KPC (carbapenemasas de clase A de Ambler) ${ }^{[13]}$.

Este es el primer reporte en la región norte del Perú de la presencia de carbapenemasas en Acinetobacter baumannii, evaluados con antibióticos sugeridos según la CLSI 2015, presentando una sensibilidad solamente al antibiótico colistina. No se encontró reportes nacionales.

Estos resultados confirman que la presencia de carbapenemasas es relativamente baja en Pseudomonas aeruginosa en comparación con Acinetobacter baumannii tanto en el Hospital Regional Lambayeque en el Perú y en el extranjero ${ }^{[8-10]}$. Aun así, la presencia de carbapenemasas tanto en Acinetobacter baumannii como Pseudomonas aeruginosa deben preocupar tanto la aparición de oxacilinasas y metalobetalactamasas porque son una amenaza sustancial a la salud que debe impulsar a las autoridades de salud formular un plan de contención, para la implementación a nivel nacional. Este plan debe asegurar la detección temprana de casos, la vigilancia permanente de brotes y una estrategia en entornos con presencia esporádica o ausencia completa de productores de Oxacilinasas y metalobetalactamasas ya que estas enzimas se pueden diseminar fácilmente en el ambiente hospitalario ${ }^{[4,14,15]}$.

Los mecanismos más utilizados por Acinetobacter baumannii y Pseudomonas aeruginosa para adquirir resistencia a los betalactámicos son: la inactivación de las drogas por las enzimas betalactamasas y la más importante es la producción de betalactamasas tipo carbapenemasas; estas enzimas son codificadas por genes que en su mayoría están localizados en elementos genéticos tales como los integrones o insertados en elementos móviles como transposones y plásmidos, y se han extendido rápidamente entre los agentes patógenos de importancia clínica, como Acinetobacter baumannii y Pseudomonas aeruginosa. $Y$ es por ello que si la resistencia a Carbapenémicos es por un mecanismo enzimático, se corre el riesgo de diseminarse rápidamente no solo a especies del mismo género sino también a las enterobacterias ${ }^{[4,14]}$.

Si bien los resultados obtenidos pertenecen a un periodo corto y la situación epidemiológica es particular para los diferentes hospitales de distintos países, es importante notar que la presencia de metalobetalactamasas en aislamientos de Pseudomonas aeruginosa y Oxacilinasas en Acinetobacter baumannii en el HRL es elevada con un $48 \%$ de las 50 cepas evaluadas. Y la presencia de estas enzimas que hidrolizan los carbapenemicos muchas veces viene acompañada de resistencia a diversas familia de antibióticos de tal manera que ha llegado a ser un fenómeno sumamente preocupante, considerado por la Organización Mundial de la Salud, por la Organización Panamericana de la Salud y los Ministerios de salud de distintas Naciones como un problema importantísimo que compromete el tratamiento de todas las enfermedades infecciosas ${ }^{[15]}$. Limitando alternativas terapéuticas y con ello una alta probabilidad de mortalidad.

Estos estudios deben realizarse en forma periódica y extenderse a más hospitales del país, debe evaluarse también la presencia de carbapenemasas en enterebacterias pues estas enzimas son fácilmente transmitidas mediante plásmidos. Idealmente se debe realizar estudios moleculares para determinar si los aislamientos productores de carbapenemasas tanto de Acinetobacter baumannii y Pseudomonas aeruginosa pertenecen a un solo linaje clonal.

En conclusión, $48 \%$ de las bacterias gram negativas no fermentadoras aisladas de los servicios de cuidados críticos del Hospital Regional Lambayeque durante diciembre 2014 a julio 2015 produjeron carbapenemasas. Todos los aislamientos de Acinetobacter baumannii produjeron carbapenemasas. La vigilancia de cepas productoras de carbapenemasas y una política para el control de la resistencia antimicrobiana son necesarias de trabajar para evitar la expansión de este problema.

Fuente de financiamiento: Los autores declaran no haber recibido ninguna financiación para la realización de este trabajo.

Conflicto de intereses: Los autores declaran no tener conflicto de intereses con la publicación de este artículo.

\section{REFERENCIAS BIBLIOGRÁFICAS}

1. Koneman E, Allen S, Janda W, Schreckenberger P, Winn W. Diagnóstico Microbiológico. 5ta ed. Buenos Aires: Medica Panamericana $\mathrm{SA}_{i} 1999$.

2. Velásquez J, Hernández $R$, Pamo $O$, Candiotti $M$, Pinedo $Y$, Sacsaquispe $R$, et al. Primer caso de carbapenemasa tipo KPC en Perú. Rev Soc Peru Med Interna. 2013;26(4):194-6.

3. Pedroza R, Cuotto W, Velázquez O, Torres L, Rodríguez L. Caracterización de plásmidos de Acinetobacter baumannii en tres centros hospitalarios. Rev Fac Med (Caracas). 2002;25(1):80-2.

4. Clinical and Laboratory Standards Institute. Performance Standards for Antimicrobial Susceptibility Testing; Twenty-First Informational Supplement. CLSI document M100-S21. Pennsylvania: Clinical and Laboratory Standards Institute; 2011.

5. Gonzales E. Metalo- $\beta$-lactamasas: ¿el fin de los $\beta$-lactámicos? Rev Peru Epidemiol. 2012;16(3):e2.

6. Pasteran F, Veliz O, Lucero C, Rapoport $M$, Ceriana $P$, Corso A. Blue Carba test (BCT) for rapid detection of carbapenemases in Gram-negatives carbapenemases. 54th Interscience Conference on Antimicrobial Agents and Chemotherapy (ICAAC), 5-9 September 2014, Washington, DC, USA. Washington: American Society for Microbiology; 2014. Abstract 963. 
7. Villegas MV, Kattan JN, Correa A, Lolans K, Guzman AM, Woodford $\mathrm{N}$, et al. Dissemination of Acinetobacter baumannii clones with OXA-23 carbapenemase in Colombian hospitals. Antimicrob Agents Chemother. 2007;51(6):2001-4.

8. Gonzales E, Vicente W, Champi R, Soto J, Flores W, Lovera M, et al. Metalo - $\beta$ - lactamasas en aislamientos clínicos de Pseudomonas aeruginosa en Lima, Perú. Rev Peru Med Exp Salud Publica. 2013;3 (2):241-5.

9. DíazJ. Detección de metalobetalactamasas (MBLs) en Pseudomonas aeruginosa resistentes a los carbapenemasas en un Hospital Nacional, en los meses de enero a octubre del año 2008. [Tesis para optar el grado académico de Magíster en Microbiología]. Lima: Facultad de Farmacia y Bioquímica, Universidad Nacional Mayor de San Marcos; 2008.

10. Perez A, Garcia P, Poggi H, Braun S, Castillo C, Ramon J, et al. Presencia de Metalo B- lactamasa en $P$. aeruginosa resistente al Imipenem. Rev Med Chile. 2008;136(4):423-32.

11. Coaguila L, Rodríguez J, Ponce R, Campos N. Infección intrahospitalaria por bacterias Gram negativas no fermentadoras en los pacientes hospitalizados en los Servicios de UCI-UCIN del Hospital Regional Lambayeque 2014 enero. Rev Exp Med. 2014; $1(2): 55-9$.
12. Menéndez J, Fernández $\mathrm{O}$, Ruiz $\mathrm{H}$, Menéndez E, Castro D. Eficacia antimicrobiana in vitro en secreciones bronquiales de pacientes con ventilación mecánica ingresados en Unidades de Cuidados Intensivos. Gac Med Espirituana. 2009;11(1):e5.

13. Radice M, Marín M, Giovanakis M, Vay C, Almuzora M, Limansky $A$, et al. Criterios de ensayo, interpretación e informe de las pruebas de sensibilidad a los antibióticos en los bacilos Gram negativos no fermentadores de importancia clínica; recomendaciones de la subcomisión de Antimicrobianos de la Sociedad Argentina de Bacteriología, Micología y Parasitología Clínicas, Asociación Argentina de Microbiología. Rev Argent Microbiol. 2011;43(2):142-4.

14. Del Valle O. Transferencia de resistencia a betalactámicos en aislados clínicos de Pseudomonas aeruginosa. [Tesis de para obtener el grado de bachiller]. Cumaná, Venezuela: Universidad de Oriente; 2012.

15. Maguiña C. Uso racional de antibióticos. 3ra Ed. Lima: Colegio Médico del Perú; 2016.

\title{
Las ediciones anteriores de Acta Médica Peruana están disponibles en:
}

\author{
www.redalyc.org
}

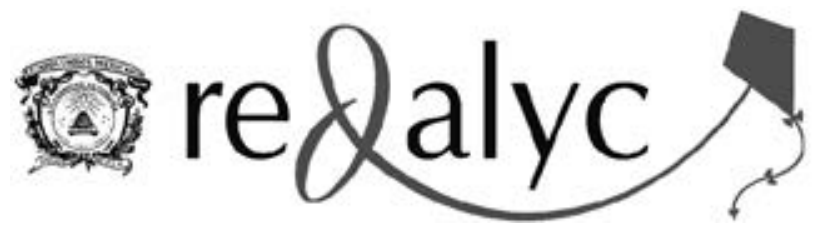

